\title{
Assessment of the Effect of Fibrillatory Waves in the Analysis of Spatial Heterogeneity of Ventricular Repolarization
}

\author{
Javier Saiz-Vivo ${ }^{1}$, Valentina D A Corino ${ }^{2}$, Massimo W Rivolta ${ }^{3}$, R Sassi ${ }^{3}$, Luca T Mainardi ${ }^{2}$ \\ ${ }^{1}$ Medtronic: Bakken Research Center, Maastricht, The Netherlands \\ ${ }^{2}$ Department of Electronics, Information and Bioengineering, Politecnico di Milano, Milan, Italy \\ ${ }^{3}$ Dipartimento di Informatica, Università degli Studi di Milano, Milan, Italy
}

\begin{abstract}
In providing proper treatment for Atrial Fibrillation (AF) patients, the clinician must establish the pattern of arrhythmia, determine associated symptoms, and asses for underlying comorbidities in order to define short- and long-term management strategies. To do so, this paper describes a preliminary study in which a novel ECG-based estimator of the standard deviation of ventricular myocytes' repolarization times $\left(s_{\vartheta}\right)$ called the $\mathcal{V}$-index was computed in simulated data, assessing whether the presence off-waves may corrupt the $\mathcal{V}$-index. The analysis was performed in simulated ECGs generated by combining synthetic bundles of 300 T-waves with seven different values of $s_{\vartheta}$, with $Q R S$ template and $50 \mathrm{f}$-waves extracted from real data. The results show a strong correlation between the calculated target $\mathcal{V}$-index ( $t \mathcal{V}$-index) and the $s_{\vartheta}(r=0.99, p$-value $<0.001)$. They also show the effect of the $f$-waves in the computation of the $\mathcal{V}$-index. The $\mathcal{V}$ index calculated with $f$-waves ( $f \mathcal{V}$-index) and after the $f$ waves were removed ( $c \mathcal{V}$-index) had highly significant differences ( $p$-value $<0.001)$ and significant differences ( $p$-value $<0.05)$ with the $t \mathcal{V}$-index for values below 53.3 $m s$, which proves the corruptive effect the $f$-waves have on the computation of the $\mathcal{V}$-index and stresses the importance of removing them before attempting further analysis.
\end{abstract}

\section{Introduction}

The progressive aging of the general population is associated with an inevitable rising in incidence of atrial fibrillation (AF) [1], which is associated to increased mortality. Even if AF is an atrial arrhythmia, it has been shown to cause ventricular repolarization remodelling [2], and to be an independent contributor to sudden cardiac death risk [3].

Several parameters related to T-wave morphology (width [4], amplitude) and duration (Tapex - Tend [5, 6] and QT dispersion [7]) have been proposed to quantify the heterogeneity of ventricular repolarization. However, when applied, they showed limitations [8, 9], have been questioned [10] or provided controversial interpretations $[9,11,12]$. Other parameters such as the one proposed by Martín-Yabra et al. focuses on the differences between pairs of T-waves when analysing AF patients under Sinus Rhythm (SR) conditions, but the index used was tailored for chronic heart failure patients only and not tested during AF conditions [13]. Having this in mind, a novel method to quantify the dispersion of myocytes' repolarization times, rooted on van Oosterom's biophysical model [14] of the ECG was derived [15]. The $\mathcal{V}$-index is an electrocardiogram (ECG)-based estimator of the standard deviation of ventricular myocytes' repolarization times $s_{\vartheta}$. The spatial dispersion of ventricular repolarization is responsible for the genesis of the T-wave on the ECG and an amplification of the heterogeneity favours the development of ventricular tachycardia/fibrillation as it creates suitable conditions for re-entry mechanisms [16, 17].

Up to now, studies involving the $\mathcal{V}$-index have been focused on patients with symptoms suggestive of non-STelevation myocardial infarction [18], assessing the effect of drugs such as moxifloxacin or sotalol [19] and studying the survival rate in patients with Chagas disease [20]. During AF the $\mathrm{T}$-waves may be corrupted by f-waves, typical of AF. Thus, when applying the $\mathcal{V}$-index to ECG during $\mathrm{AF}$, the contribution of $\mathrm{f}$-waves should be considered. This paper describes a preliminary study in which the $\mathcal{V}$-index is computed on simulated data, assessing the effect of $\mathrm{f}$-waves on the $\mathcal{V}$-index computation.

\section{Materials and Methods}

\subsection{Simulated data}

Simulated 12-lead ECGs were generated by combining synthetic T-waves with QRS complexes and f-waves extracted from real data. The ECGs were $5.57 \pm 0.41$ hours long and had a mean heart rate of $54.1 \pm 4.0 \mathrm{bpm}$.

The $\mathrm{T}$-waves were simulated using a re-implementation of the forward ECG model on which ECGSIM is based and 
provided the repolarization times across the ventricular surface [21]. The model was composed by two 3D geometries (meshes) for heart (its surface was discretized into 257 nodes) and torso, plus the bio-electrical model. The myocytes associated with a given node were lumped together and shared the same transmembrane potential (TMP). Virtual electrodes were placed on the torso mesh to resemble the standard 12-lead ECG configuration. Seven different values of $s_{\vartheta}$ were used, from 13.3 to 73.3 $\mathrm{ms}$ with step of $10 \mathrm{~ms}$ and for each one, $300 \mathrm{~T}$-waves were simulated (see Figure 1).

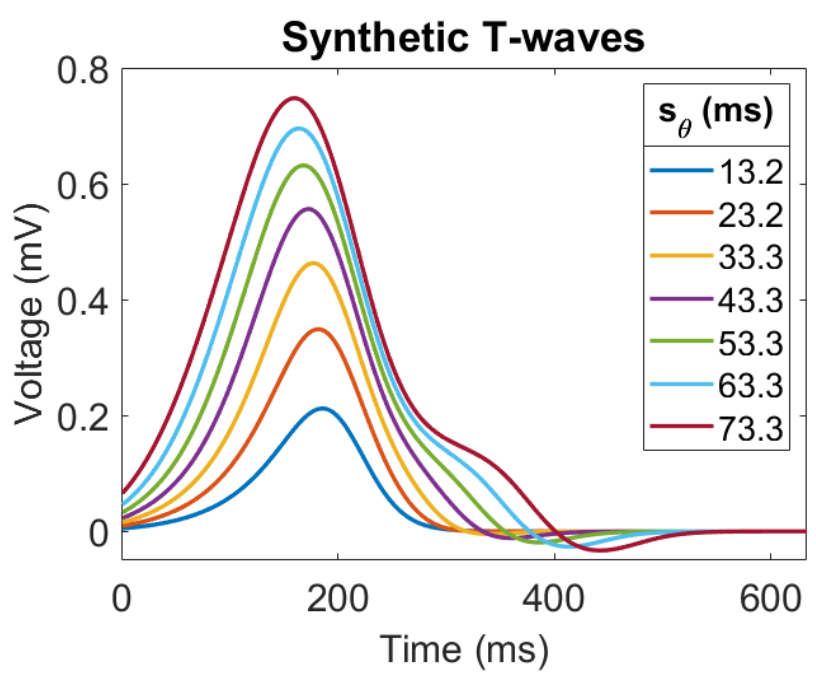

Figure 1. Median of 300 synthetic T-waves in Lead I with different values of $s_{\vartheta}(\mathrm{ms})$.

The QRS complexes from the 12 leads were extracted by locating the $\mathrm{R}$ peak and selecting a window $(-60,+100$ $\mathrm{ms}$ ) centred around it. Finally, the synthetic T-waves, the QRS complex and a $0 \mathrm{~V}$ baseline were combined with 50 ms crossfade overlap to create the resulting ECG signals.

The f-waves were then randomly extracted from 50 real AF signals using a spatiotemporal QRST cancellation technique introduced by Stridh and Sörnmo (see [22] for more details). The real $\mathrm{f}$-wave were $4.74 \pm 0.76$ hours long with mean amplitude equal to $0.10 \pm 0.05 \mathrm{mV}$ in Lead $\mathrm{I}$.

\section{2. $\quad \mathcal{V}$-index computation}

In order to calculate the $\mathcal{V}$-index, the lead factors $\boldsymbol{\omega}_{\mathbf{1}}$ and $\boldsymbol{\omega}_{2}$ have to be calculated. The method used consisted in estimating the dominant T-wave (DTW), which can be modelled by a linear equation that links the TMP to the surface potentials:

$$
\boldsymbol{\Psi}=\boldsymbol{A} \boldsymbol{D}=\boldsymbol{A}\left[\begin{array}{c}
D\left(t-\rho_{1}\right) \\
\vdots \\
D\left(t-\rho_{M}\right)
\end{array}\right]
$$

where $\boldsymbol{\Psi}$ is the vector of surface potentials and $A[L \times M]$ is a transfer matrix which is fixed for a given subject, where $\mathrm{L}$ is the number of leads and $\mathrm{M}$ the number of nodes in which the heart surface is divided. $D(t)$ represents the average shape of the repolarization phase of the TMP while $\rho_{m}$ is the repolarization time. (See [14] for more details).

Eq. 1 can also be expressed as:

$$
\boldsymbol{\Psi}(\mathrm{t}) \approx \sum_{k=1}^{N} \boldsymbol{\omega}_{\boldsymbol{k}} \frac{d^{k-1}}{d t^{k-1}} T_{d}(t)
$$

where $\boldsymbol{T}_{\boldsymbol{d}}$ is the DTW (the derivative of $D(t)$ ), $\boldsymbol{\omega}_{\boldsymbol{k}}$ is a $[L \times 1]$ vector of lead factors and $\mathrm{N}$ is the number of Taylor terms included. For computational simplicity we took $\mathrm{N}=2$ and so, Eq. 2 can be written as:

$$
\Psi(\mathrm{t}) \approx \omega_{1} T_{d}(t)+\omega_{2} \dot{T}_{d}(t)
$$

where $\boldsymbol{\omega}_{1}$ and $\boldsymbol{\omega}_{\mathbf{2}}$ are the first two lead factors and $\dot{\boldsymbol{T}}_{\boldsymbol{d}}$, the DTW's derivative (see [15] for more details on the computation of $\boldsymbol{\omega}_{1}$ and $\boldsymbol{\omega}_{2}$ ). Finally, the approximate measure of the dispersion of the ventricular myocytes' repolarization times $s_{\vartheta}$ is described by the following equation:

$$
\mathcal{V} \text {-index }=\frac{\operatorname{std}\left[\omega_{2}\right]}{\operatorname{std}\left[\omega_{1}\right]} \approx s_{\vartheta}
$$

To assess the influence of $\mathrm{f}$-waves on the computation of the $\mathcal{V}$-index, the index was computed on the simulated ECG with f-waves (fECG) as well as on the ECG where fwaves were removed (cECG). For the removal of the fwaves, a variation of the QRST cancellation algorithm was used. In this variation, once the QRST waves are cancelled in the ECG, the result which would contain the f-waves is subtracted from the original ECG, obtaining the ECG without f-waves (cECG).

\section{Results}

First, the values of the $\mathcal{V}$-index calculated before the fwaves were added ( $\mathrm{t} \mathcal{V}$-index) were compared to the standard deviation of ventricular myocytes' repolarization times $\left(s_{\vartheta}\right)$ which the synthetic T-waves were based on. In Figure $2 \mathrm{a}$, it can be appreciated that the computed $\mathcal{V}$-index is very close to the real $s_{\vartheta}$. The figure shows that the $\mathcal{V}$ index has a strong correlation with $s_{\vartheta}$ (Pearson correlation $=0.99$, p-value $<0.001)$ proving the validity of the method used to calculate the $\mathcal{V}$-index.

The $\mathcal{V}$-index was computed on the simulated ECG with added f-waves ( $\mathcal{V} \mathcal{V}$-index), and after the f-waves were cleaned $(\mathrm{c} \mathcal{V}$-index). A comparison of their values for the different $\mathrm{T}$ waves is shown in Figure $2 \mathrm{~b}$, with 


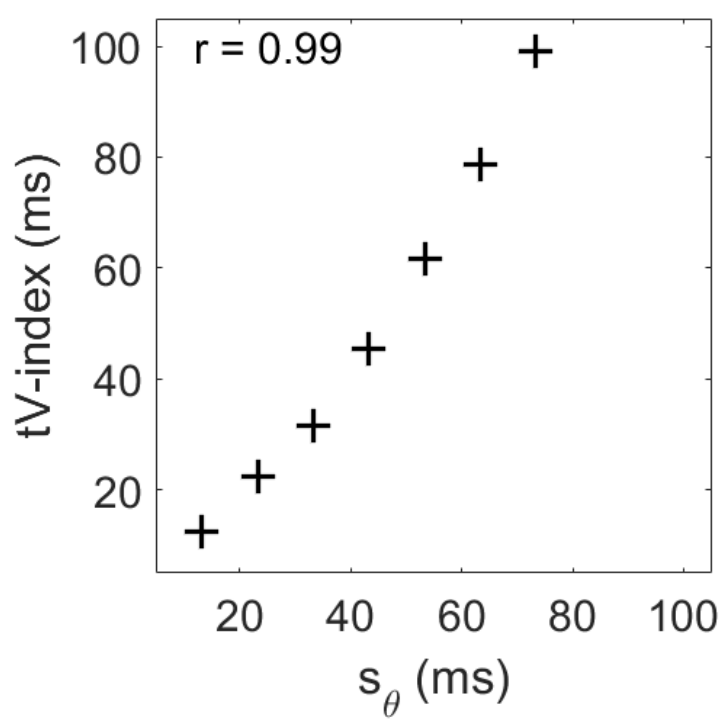

(a)

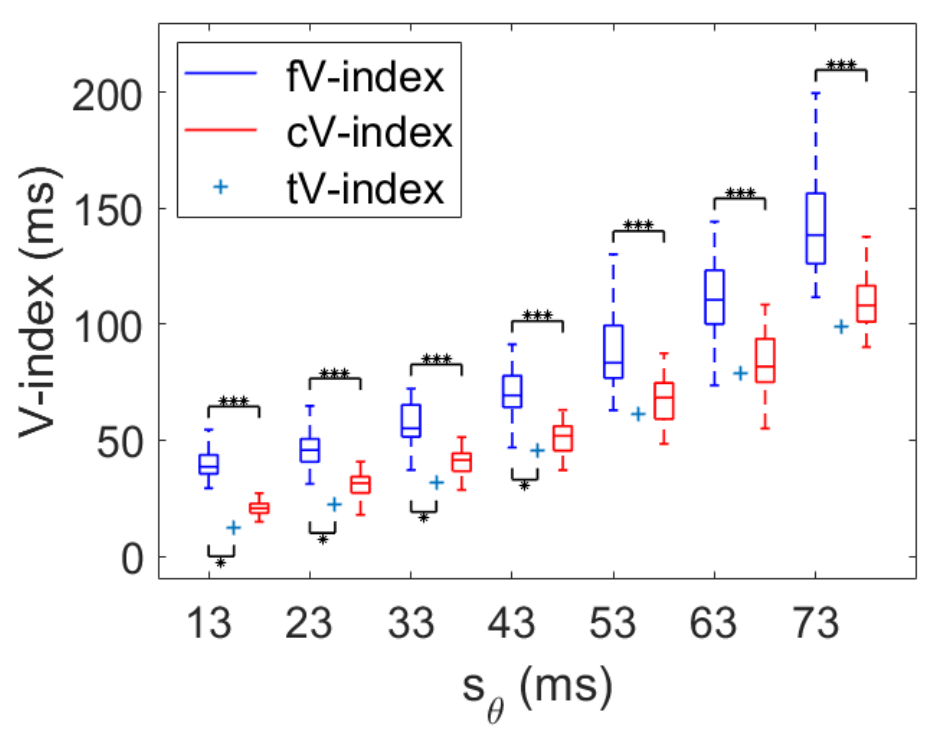

(b)

Figure 2. (a) Correlation between $\mathrm{t} \mathcal{V}$-index (ms) and $s_{\vartheta}$, (b) Comparison between $\mathcal{V}$-index with f-waves $(\mathrm{f} \mathcal{V}$-index), $\mathcal{V}$ index after $\mathrm{f}$-waves removal $(\mathrm{c} \mathcal{V}$-index $), \mathrm{t} \mathcal{V}$-index calculated.

superimposed the $\mathrm{t} \mathcal{V}$-index.

The figure shows the boxplots of the calculated $\mathcal{V}$-index when the f-waves were added ( $\mathcal{V} \mathcal{V}$-index), when the $\mathrm{f}$ waves were removed $(\mathrm{c} \mathcal{V}$-index $)$ and the target $\mathrm{t} \mathcal{V}$-index for each of the different $\mathrm{T}$-waves synthesized. It can be observed that the presence of f-waves corrupts the $\mathcal{V}$-index overestimating its value, having a highly significant difference ( $\mathrm{p}$-value $<0.001$ ) between the $\mathrm{f} \mathcal{V}$-index and $\mathrm{c} \mathcal{V}$ index. There is also a significant difference (p-value < 0.05 ) between the $f \mathcal{V}$-index and the $t \mathcal{V}$-index for values below $53.3 \mathrm{~ms}$. On the other hand, there is a non-significant difference between $\mathrm{c} \mathcal{V}$-index and $\mathrm{t} \mathcal{V}$-index for the whole range of $\mathcal{V}$-index values studied.

\section{Discussion}

Non-invasive assessment of ventricular repolarization heterogeneities during $\mathrm{AF}$ is not a straightforward task, consequence of the irregular ventricular activity present under this condition. There have been many ECG-derived markers proposed for the assessment of the heterogeneities of ventricular repolarization such as the QT interval, QT dispersion or T-wave alternans to mention a few. However, these markers require the patient to be in sinus rhythm to be properly assessed [23] which makes them less appropriate for studying AF as the properties of the AV node and atrial electrophysiology influence the highly irregular ventricular response.

In respect to QT measurements, $\mathcal{V}$-index has the advantages of being a direct estimator of the spatial heterogeneity of ventricular repolarization and of being only marginally affected by misdetection of $\mathrm{T}$-waves fiduciary points (as for instance the T-peak or T-end). However, the $\mathcal{V}$-index has previously been assessed only during SR, thus we aimed at assessing whether it is possible to quantify it during AF. As a preliminary study, we analyzed simulated data only and the results show the importance of removing the f-waves before computing the $\mathcal{V}$-index. The $\mathcal{V}$-index estimates the spatial heterogeneity of ventricular myocytes' repolarization times $s_{\vartheta}$, yielding higher values as the heterogeneity increases. The presence of $\mathrm{f}$-waves introduces a bias and the $\mathcal{V}$-index is overestimated. The findings show that the $\mathcal{V}$-index values calculated after the f-waves were removed $(\mathrm{c} \mathcal{V}$ index) are very close to the target $\mathrm{t} \mathcal{V}$-index (non-significant difference) for the range of values studied.

\section{Conclusion}

In this paper, a new methodological approach of preprocessing the ECG signal before the computation of the $\mathcal{V}$-index has been introduced. The proposed method is based on an f-wave cancellation algorithm that eliminates f-waves before the $\mathrm{T}$-waves are used to compute the $\mathcal{V}$ index. The process was tested using simulated ECG signals that were generated using synthetic $\mathrm{T}$-waves with increasing values of heterogeneity of ventricular repolarization $s_{\vartheta}$, real QRS complexes and real f-waves extracted from ECG obtained from patients with AF. It has been shown that after the f-waves were removed, the $\mathcal{V}$ index values were similar to the target $\mathrm{t} \mathcal{V}$-index values, presenting non-significant differences. In future works, the validity of the method will be tested with the computation of the $\mathcal{V}$-index during AF in clinical ECG data. 


\section{References}

[1] GBD 2015 Mortality and Causes of Death, Collaborators. "Global, regional, and national life expectancy, all-cause mortality, and cause-specific mortality for 249 causes of death, 1980-2015: a systematic analysis for the Global Burden of Disease Study 2015". Lancet. 388 (10053): 1459$1544,2016$.

[2] H.L. Tan, J.P. Smits, A. Loef, M.W. Tanck et al. "Electrocardiographic evidence of ventricular repolarization remodelling during atrial fibrillation." Europace. 2008 Jan;10(1):99-104. Epub 2007 Dec 19.

[3] L. Chen, N. Sotoodehnia, P. Buzkov et al, "Atrial fibrillation and the risk of sudden cardiac death: The atherosclerosis risk in communities study and cardiovascular health study," JAMA Internal Medicine, vol. 173, no. 1, pp. 29-35, 2013

[4] P.D. Arini, G.C. Bertrán, E.R. Valverde and P. Laguna. "Twave width as an index for quantification of ventricular repolarization dispersion: Evaluation in an isolated rabbit heart model," Biomed. Signal Process. Control, vol. 3, pp. 67-77, 2008.

[5] G.X. Yan and C. Antzelevitch, "Cellular basis for the normal T-wave and the electrocardiographic manifestations of the long-QT syndrome," Circulation, vol. 98, no. 18, pp. 1928 1936, 1998.

[6] A. Mincholé, E. Pueyo, J.F. Rodríguez, E. Zacur, M. Doblaré and P. Laguna. "Quantification of restitution dispersion from the dynamic changes of the T-wave peak to end, measured at the surface ECG," IEEE Trans. Biomed. Eng., vol. 58, no. 5, pp. 1172-1182, 2011.

[7] C.P. Day, J.M. McComb and R.W. Campbell. "QT dispersion: An indication of arrhythmia risk in patients with long QT intervals," Br. Heart J., vol. 63, no. 6, pp. 342-344, 1990.

[8] W. Zareba, "Dispersion of repolarization: Time to move beyond QT dispersion," Ann. Noninvasive Electrocardiol., vol. 5, pp. 373-381, 2000.

[9] Y. Xia, Y. Liang, O. Kongstad, Q. Liao et al. "In vivo validation of the coincidence of the peak and end of the Twave with full repolarization of the epicardium and endocardium in swine," Heart Rhythm, vol. 2, no. 2, pp. $162-169,2005$.

[10] A.J. Moss, "What resides in T-wave residuum?" J. Cardiovasc. Electrophysiol., vol. 16, no. 9, pp. 952-953, 2005.

[11] C. Antzelevitch, S. Sicouri, J.M.D. Diego, A. Burashnikov, S. Viskin et al. "Does Tpeak -Tend provide an index of transmural dispersion of repolarization?" Heart Rhythm,vol. 4, no. 8, pp. 1114-1116, 2007.

[12] T. Opthof, R. Coronel, F. J. G. Wilms-Schopman, A. N. Plotnikov et al. "Dispersion of repolarization in canine ventricle and the electrocardiographic T-wave: Tp-e interval does not reflect transmural dispersion," Heart Rhythm,vol.4, no. 3, pp. 341-348, 2007
[13] A. Martín-Yebra, I. Cygankiewicz, A. Bayés-de-Luna, P. Laguna et al. "Index of T-wave variation as a predictor of sudden cardiac death in chronic heart failure patients with atrial fibrillation," 2016 Computing in Cardiology Conference (CinC),2016, pp. 5-8.

[14] A. van Oosterom, "Genesis of the T-wave as based on an equivalent surface source model," J. Electrocardiol., vol. 34 (suppl.), pp. 217-227, 2001.

[15] R. Sassi and L. T. Mainardi. "An estimate of the dispersion of repolarization times based on a biophysical model of the ECG”. IEEE Trans Biomed Eng 2011;58:3396-3405

[16] C. S. Kuo, K. Munakata, C. P. Reddy and B. Surawicz. "Characteristics and possible mechanism of ventricular arrhythmia dependent on the dispersion of action potential durations," Circulation, vol. 67, no. 6, pp. 1356-1367, 1983.

[17] J. A. Vassallo, D. M. Cassidy, K. E. Kindwall, F. E. Marchlinski and M. E. Josephson. "Nonuniform recovery of excitability in the left ventricle," Circulation, vol. 78, no. 6, pp. 1365-1372, 1988 .

[18] R. Abächerli, R. Twerenbold, J. Boeddinghaus, T. Nestelberger et al. "Diagnostic and prognostic values of the V-index, a novel ECG marker quantifying spatial heterogeneity of ventricular repolarization, in patients with symptoms suggestive of non-ST-elevation myocardial infarction." Int J Cardiol, 2017.

[19] M. W. Rivolta, L. T. Mainardi and R. Sassi, "Quantification of ventricular repolarization heterogeneity during moxifloxacin or sotalol administration using V-index" Physiol Meas 36(4):803-811, 2015.

[20] R. Sassi, M. W. Rivolta, L. T. Mainardi, R. C. Reis et al "Spatial Repolarization Heterogeneity and Survival in Chagas Disease", Methods Inf Med, vol. 53(6), pp. 464-468, 2014.

[21] A. van Oosterom and T. F. Oostendorp, "ECGSIM: An interactive tool for studying the genesis of QRST waveforms," Heart, vol. 90, pp. 165-168, 2004.

[22] M. Stridh and L. Sörnmo. "Spatiotemporal QRST cancellation techniques for analysis of atrial fibrillation." IEEE Trans Biomed Eng., 48(1):105-11, 2001.

[23] H. J. J. Wellens, P. J. Schwartz, F. W. Lindemans et al. "Risk stratification for sudden cardiac death: current status and challenges for the future." European Heart Journal, 35(25):1642-1651, 2014

Address for correspondence:

Javier Saiz-Vivo

R\&T Department

Bakken Research Center, Medtronic

Endepolsdomein 5, Maastricht, The Netherlands

E-mail: javier.saizvivo@medtronic.com 